\title{
BMJ Open Comparison of surgical strategies in patients with spontaneous intracerebral haemorrhage: a protocol for a network meta-analysis
}

\author{
Zhiyuan Yu, Jun Zheng, Lu Ma, Rui Guo, Chao You, Hao Li
}

To cite: Yu Z, Zheng J, Ma L, et al. Comparison of surgical strategies in patients with spontaneous intracerebral haemorrhage: a protocol for a network meta-analysis. BMJ Open 2019;9:e027658. doi:10.1136/ bmjopen-2018-027658

- Prepublication history and additional material for this paper are available online. To view these files, please visit the journal online (http://dx.doi org/10.1136/bmjopen-2018027658).

Received 1 November 2018 Revised 14 March 2019 Accepted 19 June 2019

\section{Check for updates}

(c) Author(s) (or their employer(s)) 2019. Re-use permitted under CC BY-NC. No commercial re-use. See rights and permissions. Published by BMJ.

Department of Neurosurgery, West China Hospital, Sichuan University, Chengdu, China

Correspondence to Professor Hao Li; ns_liha0@126.com

\section{ABSTRACT}

Introduction Spontaneous intracerebral haemorrhage (sICH) is a severe stroke subtype. The effective therapies for patients with sICH are still unclear, and the role of surgical treatment in sICH management is still controversial. Although some large trials did not show that surgery could benefit patients with $\mathrm{sICH}$, some other studies suggested that some specific surgical strategies can have potential benefits to these patients. For a better understanding of the surgical treatment in patients with $\mathrm{sICH}$, it is necessary to conduct a network meta-analysis to compare the effects of medical treatment and different surgical methods comprehensively.

Methods and analysis This protocol has been reported following the Preferred Reporting Items for Systematic Review and Meta-Analysis Protocols. Related studies until August 2018 will be searched in the following databases: PubMed, Embase, Scopus, Web of Science, Cochrane Library, China National Knowledge Infrastructure (CNKI), VIP and Wanfang. Randomised controlled trials and non-randomised prospective studies comparing at least two different interventions in patients with sICH will be included. Quality assessment will be conducted using Cochrane Collaboration's tool or Newcastle-Ottawa Scale based on their study designs. The primary outcome will be functional outcome and the secondary outcome will be mortality. Pairwise and network meta-analysis will be conducted using STATA V.14 (StataCorp, College Station, Texas, USA). Mean ranks and the surface under the cumulative ranking curve will be used to evaluate every intervention. Statistical inconsistency assessment, subgroup analysis, sensitivity analysis and publication bias assessment will be performed.

Ethics and dissemination Ethics approval is not necessary because this study will be based on publications. The results of this study will be published in a peer-reviewed journal.

PROSPERO registration number CRD42018112239

\section{INTRODUCTION}

Spontaneous intracerebral haemorrhage ( $\mathrm{IICH})$ is the second most common and the deadliest stroke subtype. ${ }^{1}$ However, the effective therapies for patients with sICH are still unclear, and the role of surgical treatment

\section{Strengths and limitations of this study}

- This will be the first network meta-analysis that comprehensively compares different surgical strategies in patients with spontaneous intracerebral haemorrhage.

- We will include both randomised controlled studies and non-randomised prospective studies to strengthen the statistical power of this network meta-analysis.

- Quality assessment will be conducted using Cochrane Collaboration's tool or Newcastle-Ottawa Scale based on their study designs.

- Some included studies may have inferior quality and decrease the significance of the results in this network meta-analysis.

- Potentially high heterogeneity among different studies may influence the final results of this network meta-analysis.

in sICH management is still controversial. ${ }^{2}$ Theoretically, surgical removal of haematoma can reduce mass effect and secondary brain injury after sICH. ${ }^{3}$ However, the International Surgical Trial in Intracerebral Haemorrhage (STICH), a large randomised controlled trial (RCT) including 1033 patients, did not find that early surgery could benefit patients with supratentorial sICH. ${ }^{4}$ In another study including 601 patients, named STICH 2, early surgery also could not improve outcome in patients with lobar sICH. ${ }^{5}$ In these two studies, the surgical methods were not restricted and most patients received craniotomy, although endoscopy and stereotaxy were used in some cases. ${ }^{45}$ However, different surgical methods have various effects on patients with sICH.

Some other studies have been done to explore if specific surgical strategies can improve outcome in patients with sICH. Teernstra et al performed a multicentre RCT including 71 patients and found that stereotactic aspiration plus urokinase could be safely used in patients with sICH. ${ }^{6}$ Another 
important RCT, Minimally Invasive Surgery and Alteplase for ICH Evacuation, which was conducted by Hanley et $a l$, also showed that stereotactic aspiration plus alteplase was safe for haematoma removal in patients with $\mathrm{sICH}^{7}$ Vespa et al enrolled 20 patients with sICH in their RCT, named Intraoperative Stereotactic Computed Tomography-Guided Endoscopic Surgery, and showed that early endoscopic surgery was safe in patients with sICH. ${ }^{8}$ The results of these studies have suggested that some specific surgical strategies may have potential benefits to patients with sICH.

Although some meta-analyses about surgical treatment in patients with sICH have been published previously, obvious limitations exist in these studies. For example, both stereotactic aspiration and endoscopic surgery were considered as minimally invasive surgery and compared with medical treatment or craniotomy in Scaggiante $e t$ $a l$ 's study. ${ }^{9}$ In Xia $e t a l$ s study, minimally invasive surgery including stereotactic aspiration and endoscopic surgery was only compared with craniotomy. ${ }^{10}$ In another study by Ye $e t a l$, only endoscopic surgery and craniotomy were analysed. ${ }^{11}$ According to its methodology, a network meta-analysis can assess the relative effectiveness of different therapies together and estimate the rank of these therapies. ${ }^{12}$ For a better understanding of the surgical treatment in patients with sICH, it is necessary to conduct a network meta-analysis to compare the effects of medical treatment and different surgical methods comprehensively.

\section{OBJECTIVE}

This study aimed to compare the efficacy and safety of medical treatment, craniotomy, stereotactic aspiration, endoscopic surgery and decompressive craniectomy in patients with sICH using Bayesian network meta-analysis.

\section{METHODS AND ANALYSIS \\ Design}

This study will be conducted using the Bayesian network meta-analysis. The protocol has been reported following the Preferred Reporting Items for Systematic Review and Meta-Analysis Protocols ${ }^{13}$ (see online supplement 1).

\section{Registration information}

This protocol has been registered on the International Prospective Register of Systematic Review (PROSPERO). The PROSPERO registration number is CRD42018112239.

\section{Patient and public involvement}

This protocol was designed for a network meta-analysis based on the existing literature. Therefore, the patients or the public were not involved.

\section{Information source and search strategy}

We will conduct the literature search for the related RCTs and non-randomised prospective studies until August 2018 in the following databases: PubMed, Embase,
Scopus, Web of Science, Cochrane Library, CNKI, VIP and Wanfang. No restrictions on language will be set. The detailed search strategy is shown in the online supplement 2.

\section{Eligibility criteria}

Type of patients

This study will include adult patients diagnosed by CT or MRI. Studies about secondary intracerebral haemorrhage, primary intraventricular haemorrhage, subarachnoid haemorrhage or ischaemic stroke will not be included.

\section{Type of studies}

This study will include RCTs or non-randomised prospective studies. Retrospective studies, case reports, case series or reviews will not be included in this study.

\section{Type of interventions}

This study will include studies comparing at least two different interventions among the following interventions: medical treatment, craniotomy, stereotactic aspiration, endoscopic surgery and decompressive craniectomy. Studies will be excluded if there is no restriction for surgical methods.

\section{Type of outcomes}

The primary outcome will be functional outcome at the end of follow-up. Functional outcome will be dichotomised to good and poor according to the scale and threshold in each study. The secondary outcome will be mortality at the end of follow-up. The time point for outcomes will be the longest follow-up time in each study.

\section{Study selection}

After removing duplicate, titles and abstracts of all records will be screened by two authors (ZY and JZ) independently. Any record that does not meet the eligibility criteria will be removed. Full-text papers of the remaining studies will be obtained and screened by two authors independently. Only studies meeting the eligibility criteria will be finally included. If studies have duplicate data, only the study with larger sample size and longer follow-up time will be included. Any disagreement between two authors will be solved by another author (RG).

\section{Data extraction}

Based on a pre-established extraction form, two authors $(\mathrm{ZY}$ and JZ) will independently extract data from all included studies. The following information will be extracted: first author, year of publication, area, study duration, sample size, age, percentage of female, time from onset to surgery, inclusion/exclusion criteria, detailed intervention in each group, number of patients in each group, follow-up time and outcomes in each group. If some data cannot be obtained from the papers directly, we will try to contact the authors to obtain those data. Any disagreement between two authors will be solved by consensus and all data will be checked by another author (RG). 


\section{Risk of bias assessment}

The quality of all RCTs will be assessed using Cochrane Collaboration's tool. The quality of all non-randomised prospective studies will be assessed using Newcastle-Ottawa Scale. Two authors ( $\mathrm{ZY}$ and JZ) will conduct quality assessment independently and any disagreement will be solved by discussion with another author (RG).

\section{Data synthesis}

When quantitative analysis cannot be conducted, we will narratively describe the results. If quantitative analysis is feasible, all of the following statistical analyses will be conducted using STATA V.14 (StataCorp, College Station, Texas, USA).

\section{Direct comparisons of interventions}

Conventional pairwise meta-analyses between different interventions will be first conducted if at least two studies provide relevant data. DerSimonian-Laird method and random effects model will be used. ${ }^{14} I^{2}$ statistic will be used to evaluate heterogeneity among included studies. ${ }^{15}$

\section{Indirect and mixed comparisons of interventions}

Network meta-analysis will be performed with a random effects model reported in the previous study. ${ }^{16}$ Interactions among all included studies will be shown in the network geometry, and the contribution plot for the network will show the contributions of direct comparisons. ${ }^{17}$ Mean ranks and the surface under the cumulative ranking curve will be used to evaluate every intervention for both functional outcome and mortality in patients with sICH. ${ }^{18}$

\section{Statistical inconsistency assessment}

Both global and local methods will be used to assess the inconsistency between direct and indirect comparison. For global method, the design-by-treatment model will be used..$^{19}$ The loop-specific method will be adopted to assess the inconsistency locally. ${ }^{20}$

\section{Subgroup analysis and sensitivity analysis}

If possible, subgroup analyses will be performed based on age, gender, race, time from onset to surgery, Glasgow Coma Scale score, baseline haematoma volume and haematoma location. Sensitivity analysis will be performed to check the stability of the results by excluding each study.

\section{Publication bias}

Potential publication bias in the network meta-analysis will be assessed using a network funnel plot.

\section{Quality of evidence}

The evidence quality will be assessed following the Grading of Recommendations, Assessment, Development and Evaluation approach for rating the quality of treatment effect estimations from the network meta-analysis. ${ }^{21}$

\section{DISCUSSION}

This will be the first network meta-analysis that comprehensively compares different surgical strategies in patients with sICH. We will include non-randomised prospective studies to strengthen the statistical power of this network meta-analysis because the number of related randomised controlled studies is still limited. We hope the findings of this network meta-analysis can provide more information about the efficacy and safety of different surgical strategies in patients with sICH, which can help both clinical practice and study design in the future. However, limitations will still exist in this network meta-analysis. First, retrospective studies will be excluded considering their potentially low quality. However, exclusion of those studies will also increase potential publication bias. Moreover, some included studies may have inferior quality, which decreases the significance of the results in this network meta-analysis. Furthermore, potentially high heterogeneity among different studies may influence the final results of this network meta-analysis.

\section{Ethics and dissemination \\ Ethical issues}

Neither ethics approval nor patient consent is necessary because this network meta-analysis will be based on publications.

\section{Publication plan}

This protocol has been successfully registered on PROSPERO. The final results of this study will be published in a peer-reviewed journal.

Contributors $Z Y, J Z$ and $\mathrm{HL}$ are responsible for the conception of the study. ZY, JZ, $\mathrm{LM}$ and $\mathrm{HL}$ designed this protocol. $Z \mathrm{Y}$ and $\mathrm{JZ}$ tested the feasibility of this protocol. $\mathrm{ZY}, \mathrm{JZ}$ and $\mathrm{LM}$ wrote the original draft. RG, $\mathrm{CY}$ and $\mathrm{HL}$ revised the draft.

Funding This work was supported by Outstanding Subject Development 135 Project of West China Hospital, Sichuan University grant number ZY2016102 and National Natural Science Foundation of China grant number 81801186.

Competing interests None declared.

Patient consent for publication Not required.

Provenance and peer review Not commissioned; externally peer reviewed.

Open access This is an open access article distributed in accordance with the Creative Commons Attribution Non Commercial (CC BY-NC 4.0) license, which permits others to distribute, remix, adapt, build upon this work non-commercially, and license their derivative works on different terms, provided the original work is properly cited, appropriate credit is given, any changes made indicated, and the use is non-commercial. See: http://creativecommons.org/licenses/by-nc/4.0/.

\section{REFERENCES}

1. Qureshi Al, Mendelow AD, Hanley DF. Intracerebral haemorrhage. Lancet 2009;373:1632-44.

2. Hemphill JC, Greenberg SM, Anderson CS, et al. Guidelines for the management of spontaneous intracerebral hemorrhage: a guideline for Healthcare Professionals From the American Heart Association/ American Stroke Association. Stroke 2015;46:2032-60.

3. Keep RF, Hua Y, Xi G. Intracerebral haemorrhage: mechanisms of injury and therapeutic targets. Lancet Neurol 2012;11:720-31.

4. Mendelow AD, Gregson BA, Fernandes HM, et al. Early surgery versus initial conservative treatment in patients with spontaneous supratentorial intracerebral haematomas in the International Surgical Trial in Intracerebral Haemorrhage (STICH): a randomised trial. Lancet 2005;365:387-97. 
5. Mendelow AD, Gregson BA, Rowan EN, et al. Early surgery versus initial conservative treatment in patients with spontaneous supratentorial lobar intracerebral haematomas (STICH II): a randomised trial. Lancet 2013;382:397-408.

6. Teernstra OP, Evers SM, Lodder J, et al. Stereotactic treatment of intracerebral hematoma by means of a plasminogen activator: a multicenter randomized controlled trial (SICHPA). Stroke 2003;34:968-74.

7. Hanley DF, Thompson RE, Muschelli J, et al. Safety and efficacy of minimally invasive surgery plus alteplase in intracerebral haemorrhage evacuation (MISTIE): a randomised, controlled, openlabel, phase 2 trial. Lancet Neurol 2016;15:1228-37.

8. Vespa P, Hanley D, Betz J, et al. ICES (Intraoperative Stereotactic Computed Tomography-Guided Endoscopic Surgery) for Brain Hemorrhage: A Multicenter Randomized Controlled Trial. Stroke 2016;47:2749-55.

9. Scaggiante J, Zhang X, Mocco J, et al. Minimally invasive surgery for intracerebral hemorrhage. Stroke 2018;49:2612-20.

10. Xia Z, Wu X, Li J, et al. Minimally invasive surgery is superior to conventional craniotomy in patients with spontaneous supratentorial intracerebral hemorrhage: a systematic review and meta-analysis. World Neurosurg 2018;115:266-73.

11. Ye Z, Ai X, Hu X, et al. Comparison of neuroendoscopic surgery and craniotomy for supratentorial hypertensive intracerebral hemorrhage: A meta-analysis. Medicine 2017;96:e7876.

12. Bafeta A, Trinquart L, Seror R, et al. Reporting of results from network meta-analyses: methodological systematic review. BMJ (Clinical research ed). 2014;348:g 1741.
13. Moher D, Shamseer L, Clarke M, et al. Preferred reporting items for systematic review and meta-analysis protocols (PRISMA-P) 2015 statement. Syst Rev 2015;4:1.

14. DerSimonian R, Laird N. Meta-analysis in clinical trials revisited. Contemp Clin Trials 2015;45:139-45.

15. Guyatt GH, Oxman AD, Kunz R, et al. GRADE guidelines: 7 . Rating the quality of evidence-inconsistency. J Clin Epidemiol 2011;64:1294-302

16. Schmitz S, Adams R, Walsh C. Incorporating data from various trial designs into a mixed treatment comparison model. Stat Med 2013;32:2935-49.

17. Fan S, Wang D, Wu C, et al. Effects of 4 major brain protection strategies during aortic arch surgery: a protocol for a systematic review and network meta-analysis using Stata. Medicine 2018;97:e11448

18. Salanti G, Ades AE, loannidis JPA. Graphical methods and numerical summaries for presenting results from multipletreatment meta-analysis: an overview and tutorial. J Clin Epidemiol 2011;64:163-71.

19. White IR, Barrett JK, Jackson D, et al. Consistency and inconsistency in network meta-analysis: model estimation using multivariate metaregression. Res Synth Methods 2012;3:111-25.

20. Veroniki AA, Vasiliadis HS, Higgins JP, et al. Evaluation of inconsistency in networks of interventions. Int $J$ Epidemiol 2013;42:332-45.

21. Puhan MA, Schunemann HJ, Murad MH, et al. A GRADE Working Group approach for rating the quality of treatment effect estimates from network meta-analysis. BMJ 2014;349:g5630. 